\title{
Author Correction: An observed correlation between galaxy spins and initial conditions
}

\author{
Pavel Motloch (1D, Hao-Ran Yu(D, Ue-Li Pen (1D) and Yuanbo Xie
}

Correction to: Nature Astronomy https://doi.org/10.1038/s41550-020-01262-3, published online 7 December 2020.

When we loaded the external data characterizing the MaNGA and SAMI galaxies (gas velocities), we misunderstood the layout in which these data were stored, thus mixing up the row- and column-order. This effectively corresponds to transposing the arrays. This error then propagated into the three-dimensional vectors of the measured values of the spins and further into the analysis. (Catalogue entries based on the Galaxy Zoo (GZ) data were unaffected as they are not based on gas velocity data.) The analysis was repeated with the correct catalogue of measured galaxy spins. We thank $\mathrm{H}$. Jia and $\mathrm{H}$. Zhu for pointing out the problem with the first version of our catalogue of derived galaxy spins.

The correlation strength of the MaNGA and SAMI galaxy spins decreases everywhere in the investigated range of smoothing scales (light blue curve in Fig. 4). Although it is positive at smoothing scales $r \leq 3 \mathrm{Mpc} h^{-1}$, it is smaller than the correlation strength originally quoted. As a consequence, overall detection significance of the correlation is still $\sim 3 \sigma$, but decreases by $0.4-0.5 \sigma$, that is, drops from between 3 and $3.5 \sigma$ to between 2.5 and $3 \sigma$. Detection significance based on the GZ subset of the catalogue alone (red line in Fig. 5) remains qualitatively unaffected, with slight variations due to differences in the random number generator initial state when calculating the corresponding significances.

As a result of the re-analysis, the following data in Figs. 3-5 have changed: in Fig. 3, the black curve and the red and blue histograms, also the $y$-axis range; in Fig. 4, all curves; and in Fig. 5, the black and red curves. The original and corrected figures are shown below.

Consequently, in the abstract, in the sentence beginning "We find evidence", ' $\sim 3 \sigma$ ' has been changed to ' $\sim 2.7 \sigma$, and "This study presents direct evidence for..." has been changed to "This study presents evidence for...."
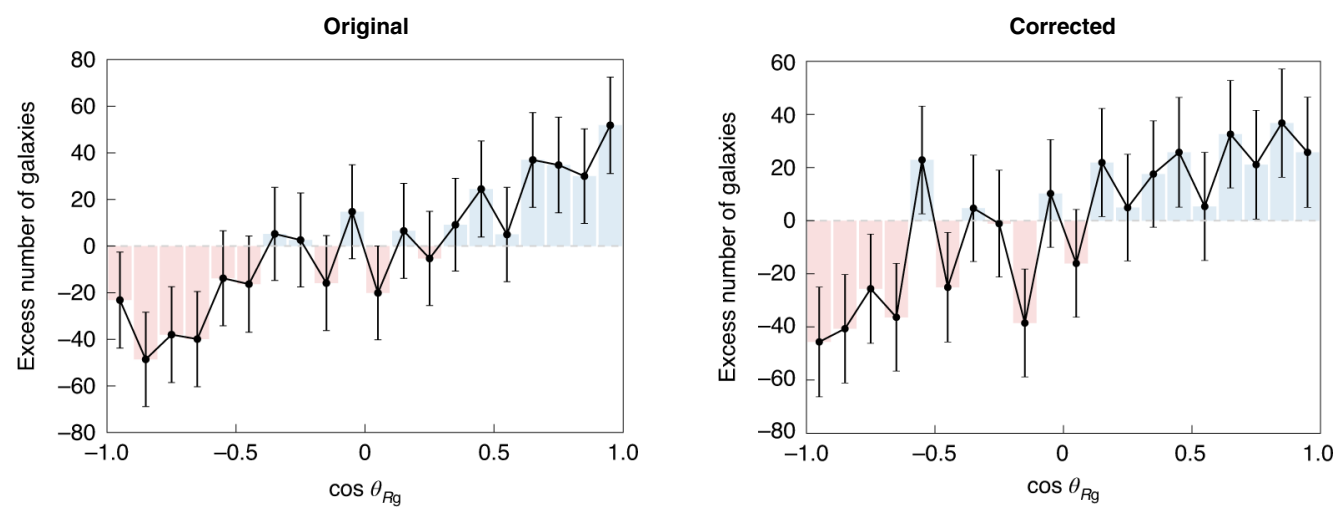

Fig. 3 | Original and Corrected.
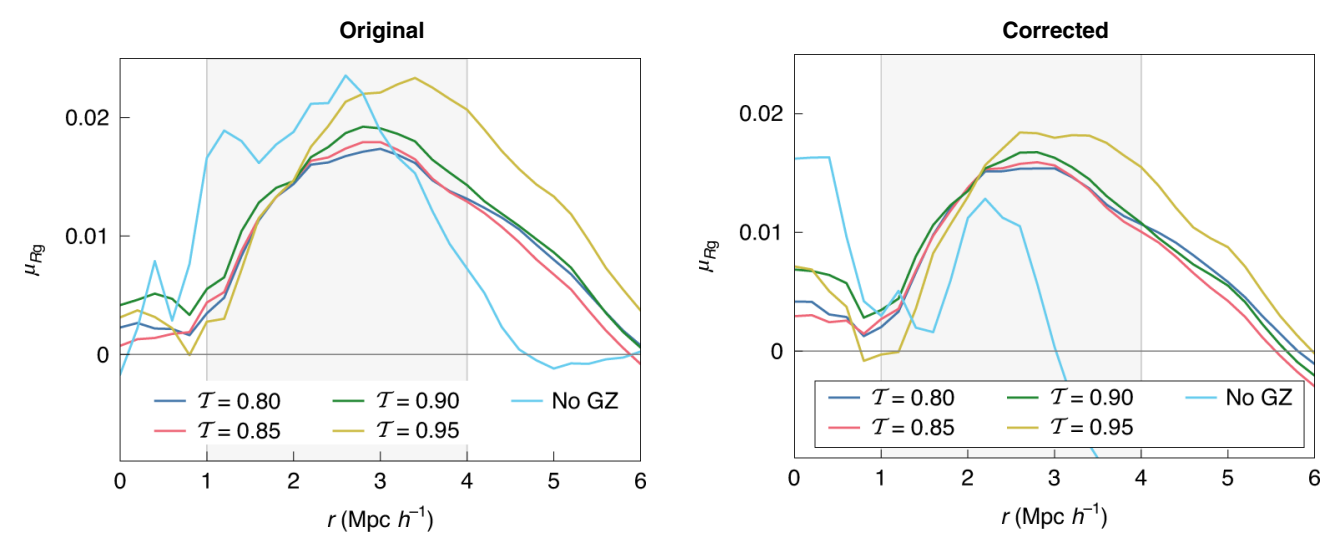

Fig. 4 | Original and Corrected. 
The following changes have also been made in the Results section: (1) in the sentence beginning "The mean value averaged over all galaxies", ' 0.017 ' now reads ' 0.016 '; (2) the sentence "In all samples, we find that $r \approx 3.0 \mathrm{Mpc} h^{-1}$ is close to optimal, with correlation strengths between 0.015 and 0.025 ." has been changed to "In all samples including GZ data, we find that $r \approx 3.0 \mathrm{Mpc} h^{-1}$ is close to optimal, with correlation strengths between 0.015 and 0.020 ."; (3) in the sentence beginning "The possibility that the $\mu_{R g}$ we see in the data", ' $3.4 \sigma$ ' now reads ' $2.9 \sigma$ '; (4) in the sentence beginning "The significance is fairly constant”, ' $95 \%$ ' has been changed to '90\%'; (5) in the sentence beginning "The detection significance", 'about 3.0 $\sigma$ ' has been changed to 'between $2.5 \sigma$ and 3.0 $\sigma$ '; (6) the sentence "MaNGA and SAMI together allow detection at only a $1.0 \sigma$ level due to the limited number of galaxies." has been changed to "MaNGA and SAMI together do not allow any significant detection due to the limited number of galaxies."

And, in the Discussion, in the sentence beginning "In this Article, we confirm”, ' $\sim 3 \sigma$ ' has been changed to ' $\sim 2.7 \sigma$ '. Finally, in the penultimate sentence of the data availability statement, 'https://doi.org/10.5281/zenodo.4075533' has been changed to 'https://doi.org/10.5281/ zenodo.4451358.

All versions of the Article have been corrected.
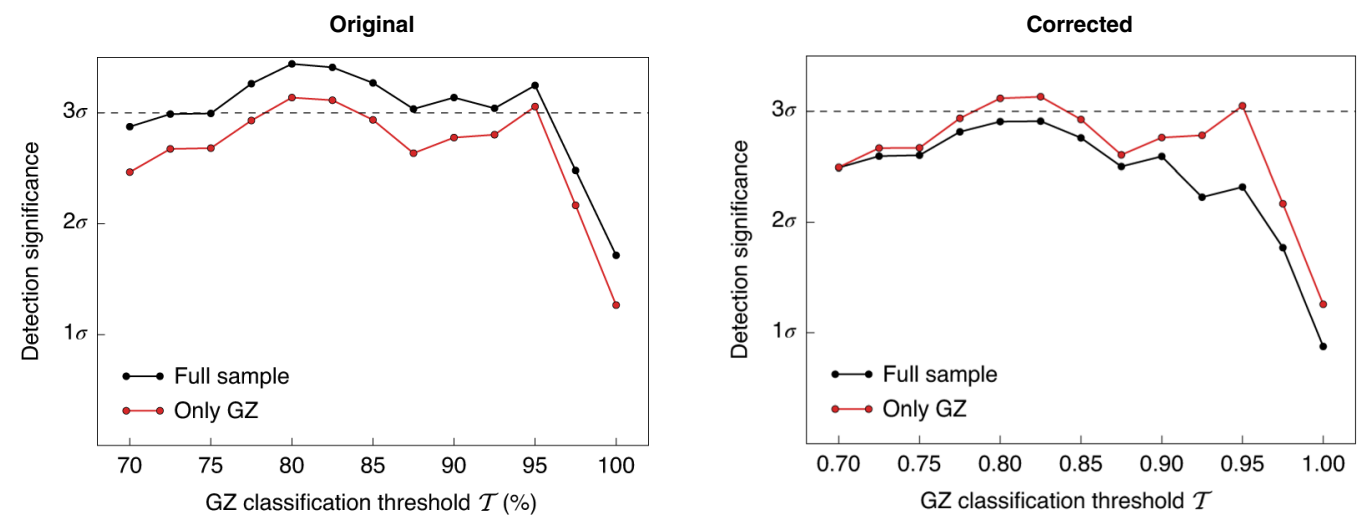

Fig. 5 | Original and Corrected.

Published online: 8 March 2021

https://doi.org/10.1038/s41550-021-01340-0

(C) The Author(s), under exclusive licence to Springer Nature Limited 2021 